\title{
Inhibition of sirtuin 2 with sulfobenzoic acid derivative AK1 is non-toxic and potentially neuroprotective in a mouse model of frontotemporal dementia
}

\author{
Tara L. Spires-Jones ${ }^{1}{ }^{*}$, Leora M. Fox ${ }^{1 \dagger}$, Anete Rozkalne ${ }^{1}$, Rose Pitstick ${ }^{2}$, George A. Carlson ${ }^{2}$ and \\ Aleksey G. Kazantsev ${ }^{1}$
}

Department of Neurology, Massachusetts General Hospital, Harvard Medical School, Charlestown, MA, USA

2 McLaughlin Research Institute, Great Falls, MT, USA

Edited by:

Tiago F. Outeiro, University of Lisbon, Portugal

\section{Reviewed by:}

Roland Seifert, Hannover Medical

School, Germany

Éva M. Szego, Georg-August

University, Germany

\section{*Correspondence:}

Tara L. Spires-Jones, Department of Neurology, Massachusetts General Hospital, Harvard Medical School, 114 16th Street, Charlestown, MA 02129, USA

e-mail: tspires@partners.org

\section{${ }^{\dagger}$ Present address:}

Leora M. Fox, Doctoral Program in Neurobiology and Behavior, Columbia University, New York, NY, USA
Tauopathies including tau-associated Frontotemporal dementia (FTD) and Alzheimer's disease are characterized pathologically by the formation of tau-containing neurofibrillary aggregates and neuronal loss, which contribute to cognitive decline. There are currently no effective treatments to prevent or slow this neural systems failure. The rTg4510 mouse model, which expresses a mutant form of the tau protein associated with FTD with Parkinsonism-17, undergoes dramatic hippocampal and cortical neuronal loss making it an ideal model to study treatments for FTD-related neuronal loss. Sirtuins are a family of proteins involved in cell survival that have the potential to modulate neuronal loss in neurodegenerative disorders. Here we tested the hypothesis that sirtuin 2 (SIRT2) inhibition would be non-toxic and prevent neurodegeneration in rTg4510 brain. In this study we delivered SIRT2 inhibitor AK1 directly to the hippocampus with an osmotic minipump and confirmed that it reached the target region both with histological assessment of delivery of a dye and with a pharmacodynamic marker, ABCA1 transcription, which was upregulated with AK1 treatment. AK1 treatment was found to be safe in wild-type mice and in the rTg4510 mouse model, and further, it provided some neuroprotection in the rTg4510 hippocampal circuitry. This study provides proof-of-concept for therapeutic benefits of SIRT2 inhibitors in both tau-associated FTD and Alzheimer's disease, and suggests that development of potent, brain permeable SIRT2 inhibitors is warranted.

Keywords: tauopathy, frontotemporal dementia, Alzheimer, sirtuin, neuroprotection

\section{INTRODUCTION}

Tauopathies are devastating conditions involving dramatic neuronal loss and resulting circuit dysfunction for which there are currently no effective treatments (Roberson, 2011). Investigation of the neuroprotective effects of sirtuin modulation may pave the way for future therapeutic studies in tauopathies and other neurodegenerative disorders which share similar mechanisms of degeneration (Spires and Hannan, 2007). Sirtuins are a family of unique $\mathrm{NAD}^{+}$-dependent enzymes, which regulate diverse cellular pathways, but are primarily involved in metabolic regulation (Guarente and Picard, 2005; Michan and Sinclair, 2007). Microtubule deacetylase sirtuin 2 (SIRT2) is a highly abundant protein in CNS, which accumulates in the aging brain (Maxwell et al., 2011). This is particularly interesting in tauopathies since tau is a microtubule binding protein and microtubule-dependent processes such as axonal transport are disrupted (Zhang et al., 2005).

In vitro evidence indicates that sirtuin modulation may be protective against amyloid toxicity in Alzheimer's disease, particularly that activating SIRT1 can be protective (Chen et al., 2005; Qin et al., 2006). Small molecule inhibitors of SIRT2 have also been found to ameliorate neurodegeneration in model systems (Outeiro et al., 2008). For example, structurally diverse selective SIRT2 inhibitors AGK2 $\left(\mathrm{IC}_{50}=3.5 \mu \mathrm{M}\right)$ and AK1 $\left(\mathrm{IC}_{50}=12.5 \mu \mathrm{M}\right)$ prevent alpha-synuclein toxicity in cell-based, and Drosophila models of Parkinson's (Outeiro et al., 2007). In Huntington's disease, both inhibitors, AGK2 and AK1, were protective against mutant polyglutamine toxicity in Drosophila and C. elegans animal models and in primary striatal neurons (LuthiCarter et al., 2010). In the latter model, the protective effects of SIRT2 inhibition were associated with reduced total cholesterol levels due to facilitated cytoplasmic retention of sterol regulatory element binding protein-2 (SREBP-2), a key transcriptional regulator of cholesterol biosynthesis genes in nuclei (Zhang et al., 2005; Luthi-Carter et al., 2010; Taylor et al., 2011). However, SIRT2 inhibition caused broad changes in transcriptional expression of metabolic genes. Cholesterol processing has also been implicated in both Alzheimer's disease (largely linked to amyloid processing) and in other tauopathies. Loss of function of the NPC1 or NPC2 genes, which are involved in trafficking cholesterol from lysosomes, has been linked to Niemann-Pick disease type $\mathrm{C}$ disease which includes tau pathology (Klunemann et al., 2002; Yu et al., 2005). Further, in the THY-Tau22 mouse model of tauopathy, long-term voluntary exercise prevented memory impairment and reduced 
hippocampal tau pathology associated with the upregulation of the NPC1 and NPC2 genes (Belarbi et al., 2011).

The promising results in efficacy of SIRT2 inhibition in two neurodegenerative diseases, the mechanistic link between the SIRT2 induced-downregulation of cholesterol biosynthesis, and the association of cholesterol dysregulation in tauopathy led to the hypothesis that SIRT2 inhibition may be neuroprotective in tauopathy. Here we assess the safety of brain delivery of the SIRT2 inhibitor AK1 in wild-type mice and in the rTg4510 transgenic model of tauopathy. The rTg4510 transgenic mouse model expresses a human tau gene with a mutation associated with familial frontotemporal dementia (FTD). These mice exhibit agerelated cognitive decline paralleled by the loss of neurons and the formation of tau-containing neurofibrillary tangles similar to those seen in Alzheimer's disease and FTD (SantaCruz et al., 2005; Spires et al., 2006; Spires-Jones et al., 2008).

In this study, we sought to test effects of SIRT2 inhibition in vivo and evaluate the safety of such an approach. Since neither of the published high efficiency SIRT2 inhibitors AGK2 nor AK1 are brain permeable, we selected a direct route for drug administration to mouse brain. Despite higher potency of SIRT2 inhibition, AGK2 has poor water solubility $(\operatorname{cLogP}=5.9)$ in contrast to AK1 $(\mathrm{cLogP}=4.0)$, making AK1 the better choice for direct administration to the brain. The high water solubility of sulfobenzoic acid derivative $\mathrm{AK} 1$ also permits robust reduction of cholesterol by this compound in striatal neurons (Luthi-Carter et al., 2010; Taylor et al., 2011).

We find that infusion of AK1 with an osmotic minipump directly into the hippocampus of mice for 5 weeks does not cause any neuronal loss in the Cornu Ammonis 1 (CA1) or dentate gyrus (DG) regions of the hippocampus compared to the untreated hemispheres or vehicle control treatment, even in the context of a tau transgenic mouse which is susceptible to neuronal loss. Furthermore, we observe a modest prevention of neuronal loss in the DG of rTg4510 mice, indicating that inhibition of SIRT2 is a safe and promising neuroprotective agent.

\section{MATERIALS AND METHODS ANIMALS AND SURGERY}

Animals used in this study were mixed gender rTg4510 mice expressing both human mutant P301L tau downstream of a tetracycline-operon-responsive element tau-P301L and an activator tetracycline-off transgene controlled by $\mathrm{Ca}^{2+}$-calmodulin kinase II promoter elements and littermate "wild-type" controls (which do not have a phenotype) expressing only the activator transgene (without tau; SantaCruz et al., 2005). Table 1 shows the numbers of animals used at each age. Each animal had an osmotic minipump (Alzet 2006, DURECT Corporation, Cupertino, CA, USA) implanted, which pumped drug or vehicle for 5 weeks before sacrifice. A further six wild-type animals were used for 1 week studies: three had a pump with $0.05 \%$ trypan blue in 5\% DMSO implanted for 1 week to verify cannula implantation coordinates and the spread of pumped solution through the hippocampus (Figure 1E) and three more received AK1 treatment for 1 week followed by gene expression analysis using qPCR. Before implantation, pumps with cannulas were assembled, filled with $50 \mu \mathrm{M}$ AK1 [3-(1-azepanylsulfonyl)-N-(3-nitrophenyl)benzamide from
Table 1 | Animals used in the study.

\begin{tabular}{llll}
\hline Genotype & Age at sacrifice (month) & Treatment & $\boldsymbol{n}$ \\
\hline Wild-type & 5.5 & AK1 & 3 \\
Wild-type & 5.5 & vehicle & 3 \\
Wild-type & 7 & AK1 & 5 \\
Wild-type & 7 & vehicle & 5 \\
rTg4510 & 5.5 & AK1 & 4 \\
rTg4510 & 5.5 & vehicle & 5 \\
rTg4510 & 7 & AK1 & 7 \\
rTg4510 & 7 & Vehicle & 5 \\
\hline
\end{tabular}

ChemBridge, diluted 1:200 in PBS from $10 \mathrm{mM}$ stock in DMSO], or vehicle (1:200 DMSO:PBS) and incubated at $37^{\circ} \mathrm{C}$ for $60 \mathrm{~h}$ to prime the pumps. To asses safety, we used the maximum concentration of AK1 $(50 \mu \mathrm{M})$ that kept the DMSO levels to a maximum of 5\%, above which DMSO can become neurotoxic (Cavas et al., 2005). Animals were anesthetized with ketamine/xylazine anesthesia (100 mg/kg ketamine, $10 \mathrm{mg} / \mathrm{kg}$ xylazine, i.p.), the pump body inserted subcutaneously over the mid-scapular area, and the cannula was implanted into the right hippocampal formation through a burr hole at coordinates -2.0 posterior to Bregma and $1.5 \mathrm{~mm}$ to the right of the midline, $1.8 \mathrm{~mm}$ deep (Figure 1C). Five weeks after pump implantation, animals were sacrificed with carbon dioxide, and brains removed and fixed in $4 \%$ paraformaldehyde for $48 \mathrm{~h}$ followed by paraffin embedding. Each cannula was tested to ensure it was not blocked and the pumps examined to ensure the compound was not remaining in the pump. Due to pump failures, group sizes are not equal. Table 1 reflects numbers of animals in each group that were analyzed (pumps worked). All animal work conforms to institutional and NIH guidelines.

\section{IMMUNOHISTOCHEMISTRY AND STEREOLOGY}

Paraffin embedded brain hemispheres were cut in sagittal orientation in $16 \mu \mathrm{m}$ sections. Every 30th section throughout the hippocampal formation was deparaffinized and stained with anti PHF-1 tau primary antibody (1:200 in 1\% milk, courtesy Dr Peter Davies), and anti mouse IgG secondary antibody conjugated to Cy3 (1:500, Jackson ImmunoResearch) and nuclei counterstained with DAPI $\left(4^{\prime}, 6\right.$-diamidino-2-phenylindole). Sections were observed on an upright Olympus BX51 fluorescence microscope with a DP70 camera and a stereology image analysis system (CAST; Olympus, Denmark). Neuronal nuclei were identified based on morphology as described previously (Spires et al., 2006; Fox et al., 2011).

Neuron densities and PHF1 positive neuron densities in the CA1 pyramidal cell layer and DG granular layer were estimated using the optical fractionator method, volumes of these regions estimated using the Cavalieri method, and total neuron number in CA1 and DG calculated as previously described (Spires et al., 2006).

\section{CELL CULTURE}

Primary cortical neurons were prepared from mouse embryos at embryonic day 15 and cultured in neurobasal media supplemented with $2 \%$ B-27 and $1 \%$ penicillin/streptomycin. At 7 days in vitro, 

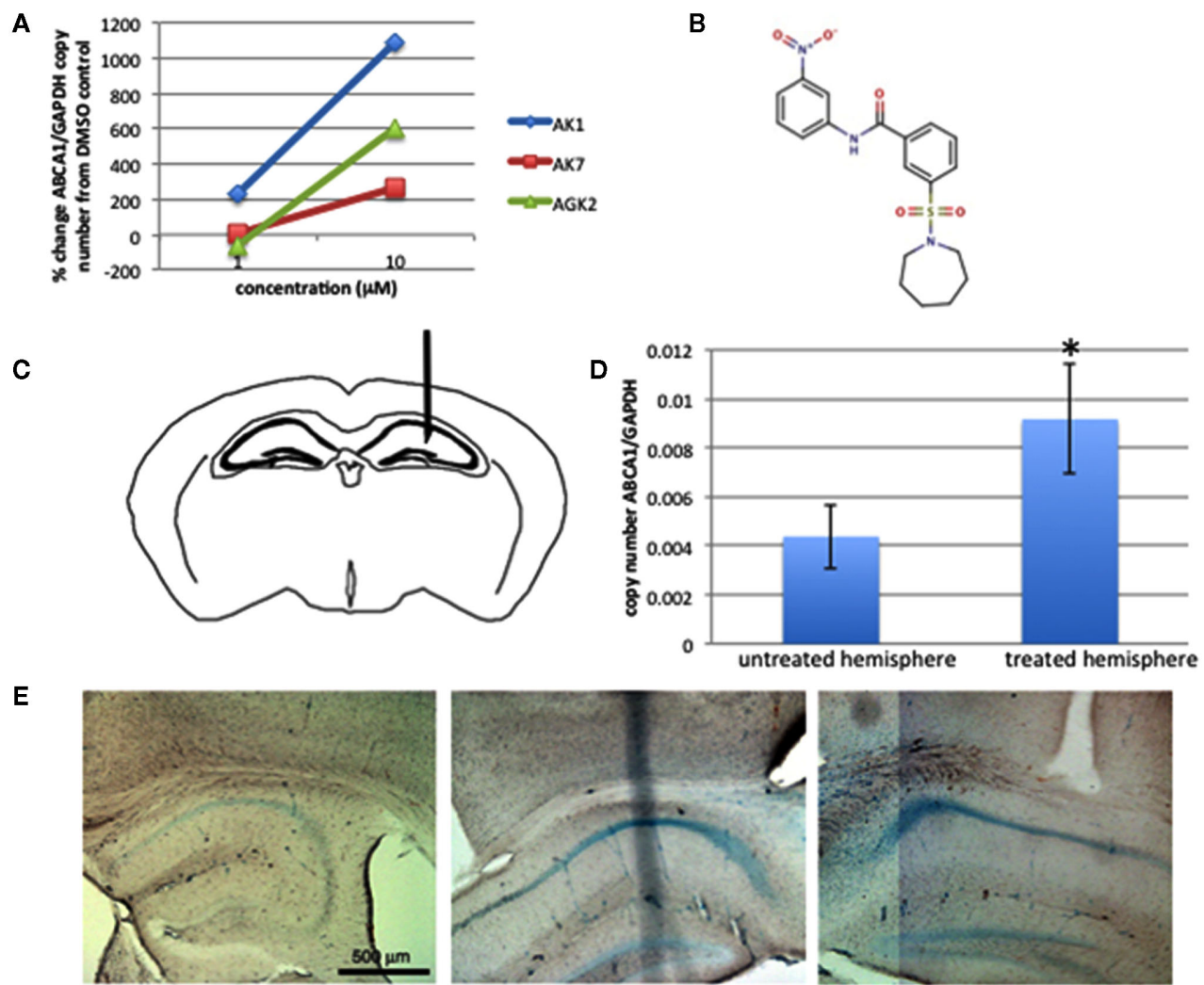

FIGURE 1 | AK1 affects ABCA1 transcription in vitro and in vivo.

Treatment of cultured primary cortical neurons with SIRT2 inhibitors AK1, $A K 7$, and AGK2 caused an increase in ABCA1 mRNA levels (A). AK1

[structure shown in (B)] was pumped into the hippocampus of mice [site shown in (C)]. As seen in cultured neurons, 1 week of treatment in

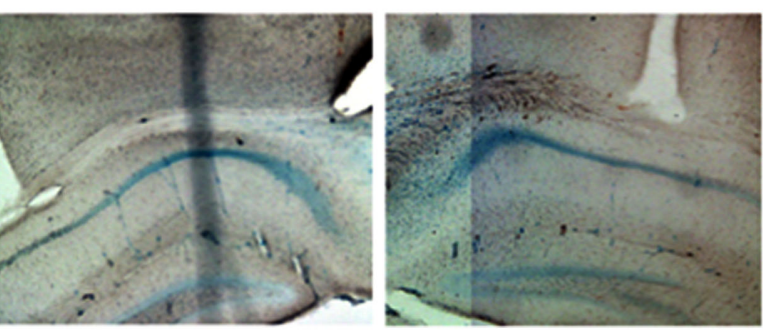

neurons were treated for $24 \mathrm{~h}$ with $1 \mu \mathrm{M}$ or $10 \mu \mathrm{M}$ AK7, AGK2, or AK1 (from ChemBridge, San Diego, CA, USA). Neurons were then harvested and homogenized for RNA extraction as described below.

\section{QUANTITATIVE PCR}

Three wild-type mice were treated with AK1 administration to the right hippocampus for 1 week. At the end of the week, mice were sacrificed, brains removed and the hemispheres frozen separately. Tissue was homogenized in RNase free lysis buffer containing $50 \mathrm{mM}$ tris buffer, $175 \mathrm{mM} \mathrm{NaCl}, 5 \mathrm{mM}$ EDTA, RNase inhibitors (Ambion AM2694), and proteinase inhibitor cocktail (Roche Complete Mini). RNA was isolated with the Stratagene Absolutely RNA Miniprep kit and cDNA generated using SuperScript reverse Transcriptase (Invitrogen). Quantitative PCR was run on an iCycler (Bio-Rad, Hercules, CA, USA) using GAPDH as a standard housekeeping gene and ABCA1 with the following primers: left, gctcaatgtcatccgtcttc; right, ctggaaacacactttccttatc.

\section{STATISTICS}

Statistical tests were run using JMP software (SAS Institute, Cary, NC, USA). Normality of data was assessed using a Shapiro-Wilk test. Non-normal data (including stereological measures) were wild-type mice increases $A B C A 1$ expression in the treated hemisphere compared to the untreated hemisphere [(D), $p<0.05]$, indicating the drug reached the brain. Trypan blue pumped for 7 days further confirms the cannula placement in the hippocampus and shows spread of pumped material through the entire hippocampal formation (E). analyzed with non-parametric tests (Kruskal-Wallis for multiple comparisons, post hoc Wilcoxon tests to compare groups). Normal data (ABCA1 expression) were analyzed with parametric statistics ( $t$-tests for two groups, ANOVA for multiple groups). $p<0.05$ is considered significant. Data are presented as mean \pm standard deviation from the mean.

\section{RESULTS}

\section{AK1 ADMINISTRATION TO THE BRAIN IS NOT NEUROTOXIC}

Previous work has shown that SIRT2 inhibition with compounds AGK2, AK1, and AK7 affects transcription of genes involved in cholesterol pathways in striatal neuronal cultures (Luthi-Carter et al., 2010; Taylor et al., 2011). We verified this in primary cortical neuronal cultures (Figure 1A), where we observed an increase in mRNA levels of ATP-binding cassette transporter ABCA1 (a known transporter of cholesterol) with SIRT2 inhibition. AK1 showed the highest efficiency in this test in our cortical cultures, and it is known to be protective in vitro against toxicity of mutant huntingtin and alpha-synuclein (Outeiro et al., 2007; Luthi-Carter et al., 2010), thus we moved forward to test the safety and efficacy of AK1 in treating tauopathy model mice. To test the safety of administering the SIRT2 inhibitor AK1 (structure shown in Figure 1B) directly to the brain, we filled osmotic 
minipumps with a solution of $50 \mu \mathrm{M}$ AK1 in PBS (with $0.5 \%$ DMSO) or vehicle and pumped the solution into the hippocampus for 5 weeks (Figure 1C). In three wild-type mice treated with AK1 for 1 week then sacrificed for molecular analysis of the brain, we confirmed that AK1 increased expression of ABCA1 in the treated versus untreated hemisphere (146.9\% higher in treated versus untreated hemisphere, Figure 1D). This indicates that AK1 may act through modulating cholesterol homeostasis as previously reported (Zhang et al., 2005; Luthi-Carter et al., 2010; Taylor et al., 2011). Further, this is a pharmacodynamic marker reporting compound activity in the brain, indicating the AK1 successfully reached the brain tissue. Tests pumping 0.05\% trypan blue for 1 week confirmed the delivery of the blue dye throughout the hippocampus (Figure 1E), further confirming the delivery of compound through the entire region of interest that we want to treat in models of tau-induced neurodegeneration.

After these preliminary studies pumping the drug into the brain for 1 week confirmed delivery of the drug to the brain and showed a pharmacodynamic response, we treated mice for 5 weeks, which is a therapeutic window long enough to observe neuronal death in mouse models of neurodegeneration. After pumping AK1 or vehicle for 5 weeks (from approximately 4 to 5.5 months of age), neuron numbers in the CA1 and DG regions of the hippocampus were estimated using stereology. In wild-type mice, we observed no neuronal loss after pumping AK1 compared to the vehicle control (Figure 2A). Comparing the treated versus untreated hemispheres in individual animals also revealed no loss in the treated side, indicating the placement of the cannula itself does not cause detectable neuronal loss (Figure 2C).

rTg4510 mice undergo neuronal loss in the hippocampus, thus we tested whether implantation of the cannula or the AK1 compound itself cause any neuronal loss in this model which is susceptible to neurodegeneration. As seen in the wild-type littermates,
5 weeks of vehicle or AK1 treatment from 4 to 5.5 months of age did not cause any neuronal loss in the CA1 or DG of rTg4510 mice (Figures 2B,D).

\section{AK1 D0ES NOT AFFECT NEUROFIBRILLARY TANGLE FORMATION}

In cellular models of Parkinson's disease, SIRT2 inhibition with AK1 prevented alpha-synuclein mediated cell death and also interestingly promoted alpha-synuclein aggregate formation (Outeiro et al., 2007). Thus we tested whether AK1 treatment from 4 to 5.5 months of age changed the number of neurofibrillary tangles in the brains of treated $\mathrm{rTg} 4510$ mice. In previous studies, we have shown that PHF1 staining in rTg4510 mice corresponds exactly to Bielchowski positive neurofibrillary tangles at these ages (Spires et al., 2006), thus PHF1 immunostaining was used to characterize neurofibrillary pathology. Stereological counts of PHF1 positive neurons show no effect of AK1 treatment on tangle numbers either when compared to vehicle treatment or when compared to the contralateral hemisphere (Figure 3), indicating that SIRT2 inhibition in vivo does not affect neurofibrillary tangle formation or stability.

\section{POTENTIAL NEUROPROTECTIVE EFFECTS OF AK1 TREATMENT}

rTg4510 mice undergo neuronal loss in the hippocampus and neocortex that is associated with behavioral deficits (SantaCruz et al., 2005; Spires et al., 2006), and neuronal loss correlates with dementia in FTD and Alzheimer's disease (DeKosky and Scheff, 1990; DeKosky et al., 1996). In control animals, there is no effect of age or treatment on the number of neurons in the DG granular cell layer $\left(1.57 \times 10^{5} \pm 0.85 \times 10^{5}\right.$ neurons per hemisphere, $p>0.05$ with treatment and age as independent variables, split by genotype). We observe a $42 \%$ loss of DG neurons between 5.5 and 7 months in vehicle treated rTg4510 mice from $1.6 \times 10^{5} \pm 0.3 \times 10^{5}$ neurons/hemisphere to

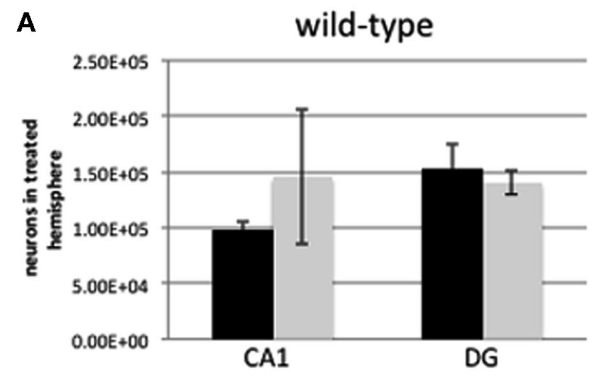

C

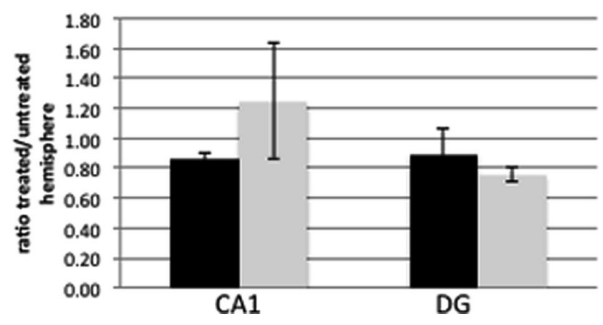

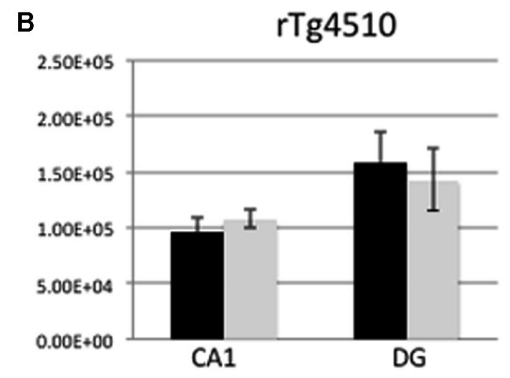

D

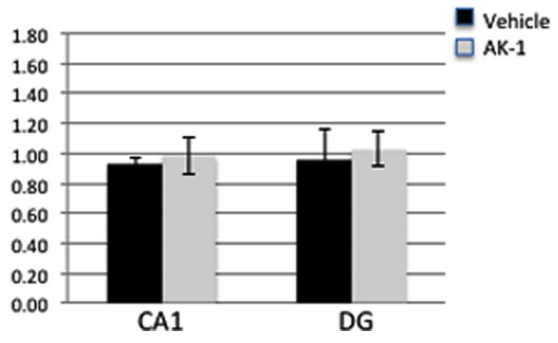

FIGURE 2 |AK1 delivery to the hippocampus is non-toxic. AK1 administration from 4 to 5.5 months of age does not cause neurodegeneration in wild-type (A) or rTg4510 hippocampus (B). Placement of the cannula in the treated hemisphere does not cause unilateral neuron loss in wild-type (C) or rTg4510 (D) hippocampus. 
$0.9 \times 10^{5} \pm 0.1 \times 10^{5}$ neurons/hemisphere $(p=0.009$, Wilcoxon test). This loss was partially prevented by AK1 treatment (Figure 4). rTg4510 mice treated with AK1 had significantly higher numbers of DG neurons than vehicle treated rTg4510 mice at 7 months ( $p=0.03$, Wilcoxon test), and they are not significantly different from control animals (control $p>0.05)$. These results indicate that $\mathrm{AK} 1$ treatment may be neuroprotective in tauopathy.

\section{DISCUSSION}

There is a pressing need to develop neuroprotective therapies for tauopathies including tau-associated FTD and Alzheimer's disease, for which there are currently no effective treatments (Roberson, 2011). One promising therapeutic avenue is manipulation of sirtuins. In particular, SIRT2 inhibition has been shown to be neuroprotective in neuronal cell culture and invertebrate animal models of Parkinson's and Huntington's diseases (Outeiro
A

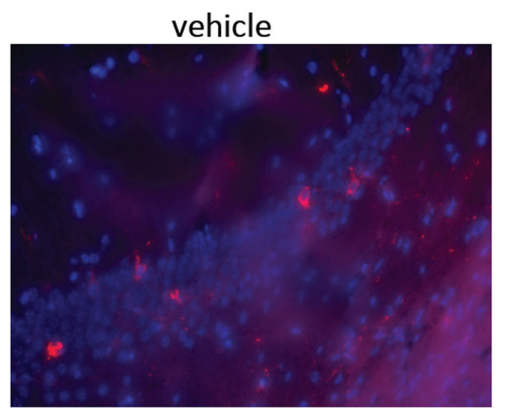

C

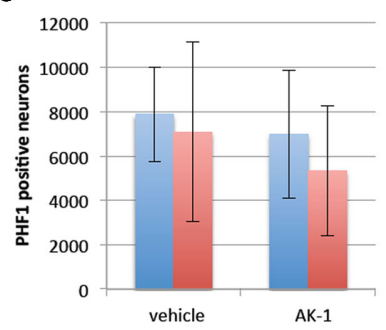

B
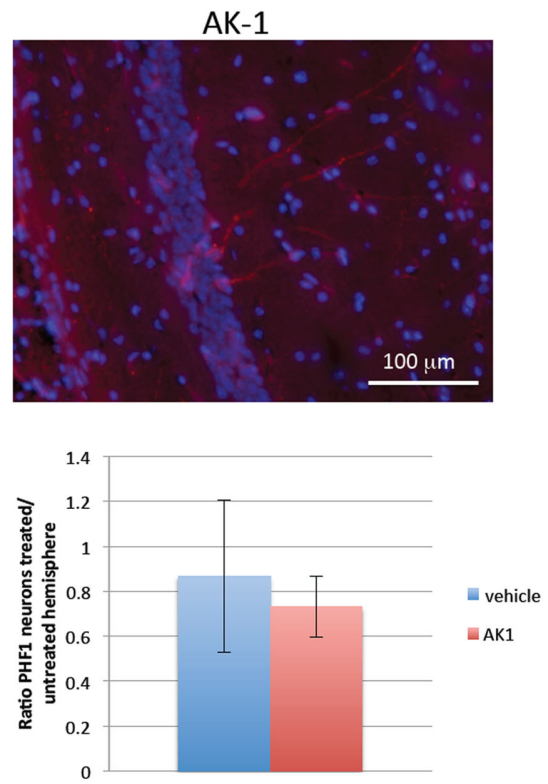

FIGURE 3 | AK1 treatment does not affect numbers of PHF1-positive neurofibrillary tangles. Micrographs from $r$ Tg4510 mice treated from 4 to 5.5 months with vehicle (A) or AK1 (B) show that both treatment groups have PHF1 positive neurofibrillary tangles (red) in the treated CA1. Quantification reveals no difference in the numbers of tangles in the treated and untreated hemispheres either when all animals are averaged (C) or when a ratio of treated/untreated hemisphere is calculated for each animal before averaging (D).
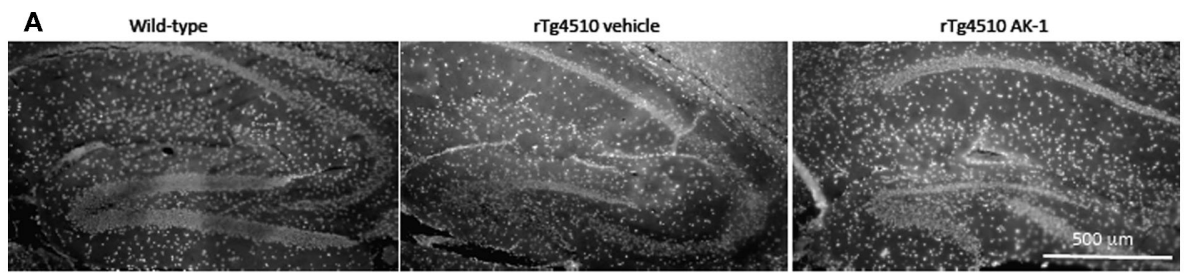

B

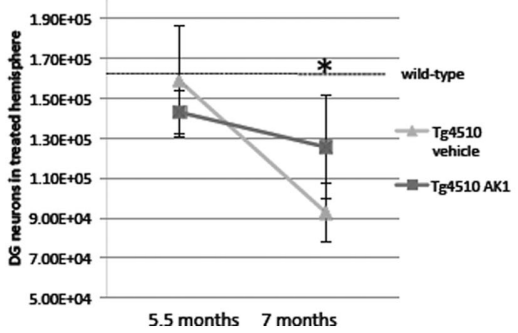

FIGURE 4 | AK1 treatment partially protects against tau-induced neurodegeneration. Micrographs of DAPI staining of the dentate gyrus (A) demonstrate the loss of neurons in rTg4510 mice compared to control mice at 7 months of age. $\mathrm{rTg} 4510$ mice treated with vehicle from 5.5 to 7 months of age underwent neuronal loss, compared both to wild-type and 5.5-month-old $r T g 4510$ animals $\left(\mathbf{B}^{*}{ }^{*} p<0.05\right)$. AK1 treatment partially prevented this loss. AK1 treated rTg4510 DG does not have significant loss compared to wild-type or 5.5-month-old rTg4510 brain. 
et al., 2007; Luthi-Carter et al., 2010). Due to the ongoing discussion and controversy on the therapeutic role of sirtuins in neurodegeneration, it was imperative to assess the properties of SIRT2 inhibition on the brain directly. While we cannot rule out off-target effects of the AK1 compound, it is the case that most marketed drugs interact with multiple targets yet mediate therapeutic effects. The purpose of this study was to assess preliminary toxicity and efficacy of a SIRT2 inhibitor belonging to a therapeutically promising structural scaffold of sulfobenzoic acid derivatives, prior to medicinal chemical optimization for drug-like properties, brain permeability, bioavailability, and bioactivity.

Tauopathies are characterized by the accumulation of aggregated tau pathology and neuronal loss. In this study, we tested whether inhibition of SIRT2 with AK1 delivery directly to the hippocampus could prevent neuronal loss in rTg4510 mice. We find that in both wild-type and $\mathrm{rTg} 4510$ brain, AK1 treatment was non-toxic to neurons (no neurons were lost with treatment from 4 to 5.5 months). Furthermore, between 5.5 and 7 months of age, neurons are lost in the DG of rTg4510 mice, and this is partially prevented by AK1 treatment. It is worth noting that the neuronal counts in this study show a phenotypic drift toward delayed neuronal loss in our rTg4510 colony with no neuronal loss in CA1 or DG between 4 and 5.5 months and no loss in CA1 between 5.5 and 7 months, whereas previous characterization of this model by our group found a steep decline in hippocampal neuron numbers in rTg4510 mice between 4 and 5.5 months of age (Spires et al., 2006). In Alzheimer's disease, neurofibrillary tangles were classically viewed as toxic since they correlate with cognitive decline, but new evidence has emerged suggesting that soluble forms of tau may be more toxic than aggregates (Spires-Jones et al., 2009). As such, it is important when developing new pharmacological targets to assess their effects both on neuronal loss and on tau aggregates. Here, we observe neuroprotection in the hippocampus without affecting neurofibrillary tangle pathology, lending support to the hypothesis that soluble tau is neurotoxic.

\section{REFERENCES}

Belarbi, K., Burnouf, S., FernandezGomez, F. J., Laurent, C., Lestavel, S., Figeac, M., Sultan, A., Troquier, L., Leboucher, A., Caillierez, R., Grosjean, M. E., Demeyer, D., Obriot, H., Brion, I., Barbot, B., Galas, M. C., Staels, B., Humez, S., Sergeant, N., Schraen-Maschke, S., Muhr-Tailleux, A., Hamdane, M., Buee, L., and Blum, D. (2011). Beneficial effects of exercise in a transgenic mouse model of Alzheimer's disease-like Tau pathology. Neurobiol. Dis. 43, 486-494.

Cavas, M., Beltran, D., and Navarro, J. F. (2005). Behavioural effects of dimethyl sulfoxide (DMSO): changes in sleep architecture in rats. Toxicol. Lett. 157, 221-232.

Chen, J., Zhou, Y., Mueller-Steiner, S., Chen, L. F., Kwon, H., Yi, S., Mucke, L., and Gan, L. (2005).

The mechanisms of tau toxicity are unclear but there is some evidence implicating altered cholesterol processing in the neurodegenerative process (Klunemann et al., 2002; Yu et al., 2005; Belarbi et al., 2011), and SIRT2 inhibition is known to modulate cholesterol biosynthesis in cultured neurons (Taylor et al., 2011). Here we confirm in vivo that the expression of ABCA1, which is a major regulator of cholesterol homeostasis, is affected in the brain with SIRT2 inhibition by AK1.

While the complexity of osmotic minipump studies resulted in the methodological limitation of a small number of animals that completed the study successfully in each group, we were still able with these small numbers to observe a modest beneficial effect of AK1 treatment and confirm that there is no overt neuronal loss induced by this compound. This study provides three important new pieces of information to the field of treatment for neurodegenerative tauopathies: (1) SIRT2 inhibition in the brain is non-toxic in wild-type and FTD model mice, (2) SIRT2 inhibition with AK1 does not affect neurofibrillary pathology in this model, and (3) AK1 treatment protects hippocampal neurons to some extent from degeneration. Osmotic minipump administration of drugs directly to the brain circumvents the blood-brain-barrier and allowed us to test the safety and efficacy of SIRT2 inhibition in vivo. However, this technique is technically difficult and not ideal for human patients. Our results suggest that the development of a potent, brain permeable compound to inhibit SIRT2 would be a good therapeutic avenue for tauopathies including FTD and Alzheimer's disease.

This study was funded by the Alzheimer's Disease Drug Discovery Foundation and the Association for Frontotemporal Degeneration, Harvard Medical School Claflin Award, NIH grants AG08487, K99AG33670, and the Alzheimer's Association Zenith Award. Renovations to McLaughlin Research Institute's Animal Resource Center were funded by major grants and donations from the M. J. Murdock Charitable Trust, Montana's Department of Commerce, the Browning-Kimball Foundation, the Oakland family, Sletten Construction, and Ian \& Nancy Davidson. We thank Prof Brad Hyman for his discussions on the project.

Guarente, L., and Picard, F. (2005) Calorie restriction - the SIR2 connection. Cell 120, 473-482.

Klunemann, H. H., Elleder, M., Kaminski, W. E., Snow, K., Peyser, J. M., O’Brien, J. F., Munoz, D., Schmitz, G., Klein, H. E., and Pendlebury, W. W. (2002). Frontal lobe atrophy due to a mutation in the cholesterol binding protein HE1/NPC2. Ann. Neurol. 52, 743-749.

Luthi-Carter, R., Taylor, D. M., Pallos, J., Lambert, E., Amore, A., Parker, A., Moffitt, H., Smith, D. L., Runne, H., Gokce, O., Kuhn, A., Xiang, Z., Maxwell, M. M., Reeves, S. A., Bates, G. P., Neri, C., Thompson, L. M., Marsh, J. L., and Kazantsev, A. G. (2010). SIRT2 inhibition achieves neuroprotection by decreasing sterol biosynthesis. Proc. Natl. Acad. Sci. U.S.A. 107, 7927-7932.
Maxwell, M. M., Tomkinson, E. M., Nobles, J., Wizeman, J. W., Amore, A. M., Quinti, L., Chopra, V., Hersch, S. M., and Kazantsev, A. G. (2011). The Sirtuin 2 microtubule deacetylase is an abundant neuronal protein that accumulates in the aging CNS. Hum. Mol. Genet. 20, 3986-3996.

Michan, S., and Sinclair, D. (2007). Sirtuins in mammals: insights into their biological function. Biochem. J. 404, 1-13.

Outeiro, T. F., Kontopoulos, E., Altmann, S. M., Kufareva, I., Strathearn, K. E., Amore, A. M., Volk, C. B., Maxwell, M. M., Rochet, J. C. Mclean, P. J., Young, A. B., Abagyan, R., Feany, M. B., Hyman, B. T., and Kazantsev, A. G. (2007). Sirtuin 2 inhibitors rescue alpha-synucleinmediated toxicity in models of Parkinson's disease. Science 317, 516-519. 
Outeiro, T. F., Marques, O., and Kazantsev, A. (2008). Therapeutic role of sirtuins in neurodegenerative disease. Biochim. Biophys. Acta 1782, 363-369.

Qin, W., Yang, T., Ho, L., Zhao, Z., Wang, J., Chen, L., Zhao, W., Thiyagarajan, M., Macgrogan, D., Rodgers, J. T., Puigserver, P., Sadoshima, J., Deng, H., Pedrini, S., Gandy, S., Sauve, A. A., and Pasinetti, G. M. (2006). Neuronal SIRT1 activation as a novel mechanism underlying the prevention of Alzheimer disease amyloid neuropathology by calorie restriction. J. Biol. Chem. 281, 21745-21754.

Roberson, E. D. (2011). Contemporary approaches to Alzheimer's disease and frontotemporal dementia. Methods Mol. Biol. 670, 1-9.

SantaCruz, K., Lewis, J., Spires, T., Paulson, J., Kotilinek, L., Ingelsson, M., Guimaraes, A., Deture, M., Ramsden, M., Mcgowan, E., Forster, C., Yue, M., Orne, J., Janus, C., Mariash, A., Kuskowski, M., Hyman, B., Hutton, M., and Ashe, K. H. (2005). Tau suppression in a neurodegenerative mouse model improves memory function. Science 309, 476-481.

Spires, T. L., and Hannan, A. J. (2007). Molecular mechanisms mediating pathological plasticity in Huntington's disease and Alzheimer's disease. J. Neurochem. 100, 874-882.

Spires, T. L., Orne, J. D., SantaCruz, K., Pitstick, R., Carlson, G. A., Ashe, K. H., and Hyman, B. T. (2006). Region-specific dissociation of neuronal loss and neurofibrillary pathology in a mouse model of tauopathy. Am. J. Pathol. 168, 1598-1607.

Spires-Jones, T. L., De Calignon, A., Matsui, T., Zehr, C., Pitstick, R., Wu, H. Y., Osetek, J. D., Jones, P. B., Bacskai, B. J., Feany, M. B., Carlson, G. A., Ashe, K. H., Lewis, J., and Hyman, B. T. (2008). In vivo imaging reveals dissociation between caspase activation and acute neuronal death in tangle-bearing neurons. J. Neurosci. 28, 862-867.

Spires-Jones, T. L., Stoothoff, W. H., De Calignon, A., Jones, P. B., and Hyman, B. T. (2009). Tau pathophysiology in neurodegeneration: a tangled issue. Trends Neurosci. 32, 150-159.
Taylor, D. M., Balabadra, U., Xiang, Z., Woodman, B., Meade, S., Amore, A., Maxwell, M. M., Reeves, S., Bates, G. P., Luthi-Carter, R., Lowden, P. A., and Kazantsev, A. G. (2011). A brainpermeable small molecule reduces neuronal cholesterol by inhibiting activity of sirtuin 2 deacetylase. ACS Chem. Biol. 6, 540-546.

Yu, W., Ko, M., Yanagisawa, K., and Michikawa, M. (2005). Neurodegeneration in heterozygous Niemann-Pick type C1 (NPC1) mouse: implication of heterozygous NPC1 mutations being a risk for tauopathy. J. Biol. Chem. 280, 27296-27302.

Zhang, B., Maiti, A., Shively, S., Lakhani, F., Mcdonald-Jones, G., Bruce, J., Lee, E. B., Xie, S. X., Joyce, S., Li, C., Toleikis, P. M., Lee, V. M., and Trojanowski, J. Q. (2005). Microtubulebinding drugs offset tau sequestration by stabilizing microtubules and reversing fast axonal transport deficits in a tauopathy model. Proc. Natl. Acad. Sci. U.S.A. 102, 227-231.

Conflict of Interest Statement: The authors declare that the research was conducted in the absence of any commercial or financial relationships that could be construed as a potential conflict of interest.

Received: 30 January 2012; paperpending published: 18 February 2012; accepted: 24 February 2012; published online: 12 March 2012.

Citation: Spires-Jones TL, Fox LM, Rozkalne A, Pitstick R, Carlson GA and Kazantsev AG (2012) Inhibition of sirtuin 2 with sulfobenzoic acid derivative $A K 1$ is non-toxic and potentially neuroprotective in a mouse model of frontotemporal dementia. Front. Pharmacol. 3:42. doi: 10.3389/fphar.2012.00042

This article was submitted to Frontiers in Experimental Pharmacology and Drug Discovery, a specialty of Frontiers in Pharmacology.

Copyright (C) 2012 Spires-Jones, Fox, Rozkalne, Pitstick, Carlson and Kazantsev. This is an open-access article distributed under the terms of the Creative Commons Attribution Non Commercial License, which permits non-commercial use, distribution, and reproduction in other forums, provided the original authors and source are credited. 